\title{
Paediatric crossword puzzle - 20 (Answers)
}

Manouri P Senanayake',

Sri Lanka Journal of Child Health; 42(4): 228

\begin{tabular}{|l|l|l|l|l|l|l|l|l|l|l|l|l|l|l|}
\hline & ${ }^{1} \mathrm{D}$ & $\mathrm{I}$ & ${ }^{2} \mathrm{~A}$ & $\mathrm{~B}$ & $\mathrm{E}$ & $\mathrm{T}$ & $\mathrm{E}$ & ${ }^{3} \mathrm{~S}$ & & & ${ }^{4} \mathrm{D}$ & $\mathrm{C}$ & $\mathrm{D}$ & \\
\hline $\mathrm{V}$ & & & $\mathrm{L}$ & & & & & $\mathrm{R}$ & & & ${ }^{7} \mathrm{D}$ & & ${ }^{8} \mathrm{~W}$ & \\
\hline $\mathrm{E}$ & & ${ }^{6} \mathrm{P}$ & & & ${ }^{10} \mathrm{~W}$ & ${ }^{11} \mathrm{~A}$ & $\mathrm{I}$ & $\mathrm{S}$ & ${ }^{12} \mathrm{~T}$ & & ${ }^{13} \mathrm{I}$ & $\mathrm{g}$ & $\mathrm{A}$ & \\
\hline${ }^{14} \mathrm{~V}$ & $\mathrm{U}$ & $\mathrm{R}$ & & & & $\mathrm{L}$ & & & $\mathrm{R}$ & & $\mathrm{R}$ & & $\mathrm{Y}$ & \\
\hline $\mathrm{A}$ & & $\mathrm{E}$ & & & & $\mathrm{B}$ & & ${ }^{15} \mathrm{C}$ & $\mathrm{O}$ & $\mathrm{R}$ & $\mathrm{O}$ & $\mathrm{N}$ & $\mathrm{A}$ & \\
\hline $\mathrm{M}$ & & ${ }^{16} \mathrm{D}$ & $\mathrm{E}$ & ${ }^{17} \mathrm{~N}$ & $\mathrm{G}$ & $\mathrm{U}$ & $\mathrm{E}$ & & $\mathrm{P}$ & & $\mathrm{F}$ & & $\mathrm{M}$ & \\
\hline${ }^{18} \mathrm{I}$ & $\mathrm{M}$ & $\mathrm{N}$ & & $\mathrm{G}$ & & $\mathrm{M}$ & & & $\mathrm{O}$ & & $\mathrm{I}$ & & ${ }^{19} \mathrm{~B}$ & $\mathrm{~T}$ \\
\hline $\mathrm{S}$ & & $\mathrm{O}$ & & $\mathrm{A}$ & & $\mathrm{I}$ & & & $\mathrm{N}$ & & ${ }^{20} \mathrm{~L}$ & $\mathrm{O}$ & $\mathrm{A}$ & \\
\hline $\mathrm{O}$ & & ${ }^{21} \mathrm{~S}$ & ${ }^{22} \mathrm{~A}$ & $\mathrm{~L}$ & ${ }^{23} \mathrm{I}$ & $\mathrm{N}$ & $\mathrm{E}$ & & ${ }^{24} \mathrm{I}$ & $\mathrm{H}$ & $\mathrm{A}$ & & & ${ }^{25} \mathrm{~W}$ \\
\hline $\mathrm{L}$ & & & $\mathrm{Z}$ & & $\mathrm{L}$ & & & & $\mathrm{N}$ & & ${ }^{26} \mathrm{R}$ & $\mathrm{O}$ & $\mathrm{T}$ & $\mathrm{H}$ \\
\hline${ }^{27} \mathrm{E}$ & ${ }^{28} \mathrm{M}$ & $\mathrm{B}$ & $\mathrm{O}$ & $\mathrm{L}$ & $\mathrm{I}$ & $\mathrm{S}$ & ${ }^{29} \mathrm{M}$ & & & & $\mathrm{I}$ & & & $\mathrm{E}$ \\
\hline & $\mathrm{R}$ & & $\mathrm{L}$ & & $\mathrm{A}$ & & $\mathrm{E}$ & & ${ }^{30} \mathrm{D}$ & ${ }^{31} \mathrm{E}$ & $\mathrm{A}$ & $\mathrm{D}$ & $\mathrm{L}$ & $\mathrm{Y}$ \\
\hline
\end{tabular}

${ }^{1}$ Professor in Paediatrics, Faculty of Medicine, University of Colombo 